\title{
Quaderni
}

QUADERN I Communication, technologies, pouvoir

\section{Entre personnalisation et responsabilisation : le travail relationnel "sous pression" dans les soins oncologiques}

Hélène Marche

\section{(2) OpenEdition \\ Journals \\ Édition électronique \\ URL : http://journals.openedition.org/quaderni/743 \\ DOI : 10.4000/quaderni.743 \\ ISSN : 2105-2956 \\ Éditeur \\ Les éditions de la Maison des sciences de l'Homme}

\section{Édition imprimée}

Date de publication : 5 octobre 2013

Pagination : 55-66

Référence électronique

Hélène Marche, "Entre personnalisation et responsabilisation : le travail relationnel "sous pression" dans les soins oncologiques », Quaderni [En ligne], 82 | Automne 2013, mis en ligne le 05 octobre 2015, consulté le 01 mai 2019. URL : http://journals.openedition.org/quaderni/743; DOI : 10.4000/ quaderni.743 


\section{$D$ ossier}

\section{Entre}

personnalisation et responsabilisation :

le travail

relationnel

"sous pression"

dans les soins

oncologiques

\section{Hélène Marche}

Docteure en sociologie, chercheure associée au laboratoire Espaces et Sociétés (ESO - Rennes UMR 6590)
En cancérologie, l'enjeu de responsabiliser les individus à l'égard de leur santé (ou de leur maladie) se double de celui de repérer et d'accompagner des formes de vulnérabilité (corporelle, psychique et sociale) auxquelles ils sont réellement ou supposément confrontés. Tout en étant encadrés par des réformes récentes ou en cours ${ }^{1}$, ces enjeux impliquent de la part des professionnels un travail de qualification des capacités d'action des malades qui se complexifie dans les situations où plusieurs acteurs sont amenés à revendiquer ou à reconnaître ces capacités à agir, ou encore lorsque des conflits latents entre malades, proches et professionnels tendent à s' accroître ${ }^{2}$, ce qui est le cas lorsque les activités médicales s'orientent d'une prise en charge curative vers une prise en charge palliative ${ }^{3}$. Les médecins et soignants sont alors confrontés à plusieurs types d'incertitude. À l'incertitude médicale liée à l'évolution de la pathologie cancéreuse et aux connaissances dont disposent les professionnels pour en gérer le cours ${ }^{4}$, s'ajoute une incertitude relationnelle, celle-ci se rapportant aux postures adoptées par le patient en tant que « sujet » et à la nature des relations engagées dans la situation de maladie.

Des conditions structurelles tendent à accroître ce type d'incertitude. Dans un contexte de rationalisation et de technicisation des soins oncologiques, les trajectoires du cancer se déploient dans une pluralité de lieux (différents établissements et services, domicile du patient) et impliquent une pluralité d'acteurs, professionnels et non-professionnels. Au sein des services de cancérologie, le temps dont disposent les soignants pour accompagner les malades est soumis à de fortes pressions organisationnelles (libérer des chambres pour accueillir de nouveaux patients, lourdeur de cer- 
tains soins techniques, etc.). Le travail de soin est alors susceptible d'être délégué à l'entourage du malade, ce qui confronte les soignants à une incertitude quant à la façon dont ces derniers vont s'en saisir (adéquation ou non aux conceptions professionnelles).

Partant, ce texte vise à éclairer les variations du travail relationnel des professionnels à destination des malades atteints de cancer avancé dans un contexte de réduction des séjours hospitaliers et de rationalisation des soins. Dès l'entrée du malade dans un service, la question est posée par les professionnels : faut-il maintenir le patient hospitalisé, envisager un retour à domicile ou un transfert vers un autre établissement, à plus ou moins long terme ${ }^{5}$ ? Comment le patient se positionne-t-il face à ces différentes alternatives, et comment combiner sa volonté aux possibilités qui sont offertes?

\section{Méthodologie}

L'enquête ethnographique a été principalement menée dans un service de soins continus/soins palliatifs d'un établissement dédié au traitement du cancer, de février 2011 à mai 2011 ${ }^{6}$. Le service observé comporte 26 lits répartis sur trois secteurs (12 chambres simples, sept chambres doubles). Huit chambres simples sont destinées à l'accueil de malades identifiés comme relevant de soins palliatifs ${ }^{7}$. Les médecins référents des patients hospitalisés travaillant dans l'établissement assurent également les visites. Le service travaille en liaison avec une équipe d'hospitalisation à domicile (HAD).

Des observations ont également été effectuées lors de réunions rassemblant des professionnels des soins palliatifs intervenant à l'échelle départementale (services de cancérologie, EMSP, HAD, USP, etc.), celles-ci visant à orienter les malades vers les lieux de soins désignés comme appropriés à leur situation, ou bien à améliorer les conditions de leur prise en charge (gestion de la douleur, résolution de conflits avec le malade et/ou ses proches, etc.).

À partir de ces observations et d'entretiens compréhensifs menés auprès de malades, de proches et de professionnels ${ }^{8}$, l'analyse des données a consisté à décrire la façon dont les trajectoires des malades sont négociées entre différents acteurs, professionnels et profanes, selon les situations de maladie et selon les contextes de soins ${ }^{9}$. La construction d'études de cas a permis de mettre en perspective les différentes trajectoires de soins observées et de souligner les points de correspondance ou de contraste qui apparaissent entre elles. Deux études de cas, concernant des patients atteints d'un sarcome avancé, seront mises en perspective. Avant cela, il est nécessaire de souligner les modalités du travail relationnel des professionnels et d'indiquer leurs spécificités autour des enjeux d'accueillir les malades dans le service, de les y maintenir ou de les transférer vers un autre lieu de soin.

\section{Le travail relationnel face à la dimension spatio-temporelle des trajectoires du cancer}

L'《 esprit de soin » destiné aux malades atteints de cancer avancé implique « (...) une préoccupation très importante sur la personne malade, sur sa famille, son environnement, sur son projet. Et une attention, effectivement, un petit peu à tout ce 
qui pouvait la concerner, même en-dehors de la maladie elle-même ». (Médecin, 51 ans, service de cancérologie/soins palliatifs).

Si les professionnels considèrent le malade en tant que «sujet », la conception qu'ils en ont prend différents sens. Irréductible à un « homo clausus $»^{10}$ considéré sous le seul angle de sa subjectivité ou de son intériorité, ou encore à la figure du «patient-sentinelle $»^{11}$, le « sujet de soin » est une norme sociale et morale que les professionnels mobilisent pour orienter le travail relationnel à destination du malade et de son entourage. Cette norme est flexible, dans le sens où elle tend à varier selon les contextes et les séquences des trajectoires du cancer (temps des traitements, récidive, arrêt des traitements curatifs, etc.). Elle varie également selon les positions et expériences professionnelles ${ }^{12}$. Il est possible de dégager quatre façons dont les soignants « travaillent » la relation au patient : la responsabilisation, pour laquelle le malade est considéré comme un «acteur de sa propre santé », la personnalisation, dans laquelle le malade est considéré comme un sujet « sensible » et disposant d'une conscience propre de sa situation, la protection, où la figure du malade qui prédomine est celle d'un sujet vulnérable ou fragile et enfin la réparation, où la conception du malade est celle d'un sujet déstructuré, dont la relation à lui-même et aux autres a été fragilisée ou rom$p^{13}{ }^{13}$. En fonction des situations auxquelles ils se confrontent, les soignants font appel à ces diverses conceptions du « sujet de soin » en vue d'adapter le travail relationnel.

Au sein des services observés, c'est d'abord le travail de personnalisation qui est valorisé par l'équipe. Dans ce modèle, la progression de la maladie est considérée comme favorisant la prise de conscience du malade vis-à-vis d'une nouvelle réalité : «Parce qu'il me semble que quelqu'un qui va mal le sent forcément de façon assez intime dans son corps. Et que, très souvent lorsqu'on l'invite à préciser comment il ressent les choses à l'intérieur, c'est une façon un petit peu de les inviter à partager un peu leurs inquiétudes. Du coup, pour pouvoir soit les partager, soit au contraire les minimiser » (Médecin, 51 ans, service de cancérologie).

Ce faisant, la norme est celle de respecter le point de vue du malade et de ne pas lui imposer un pronostic qu'il ne serait pas " prêt à entendre". Ce modèle se distingue ainsi de celui de la responsabilisation, qui consiste à transmettre au malade les informations médicales afin qu'il se positionne et participe activement aux propositions de l'équipe. Il se distingue également du modèle de la protection, qui vise quant à lui à maintenir le patient à distance des informations liées au pronostic. Cependant, en termes organisationnels, le travail de personnalisation « prend du temps » aux professionnels, puisqu'il s'agit de réitérer les rencontres auprès du malade, de lui « tendre des perches » ou de l'aider à formuler ses inquiétudes afin qu'il puisse progressivement accéder à sa conscience propre. Il s'agit également d'un modèle qui « prend de la place » dans le service, puisqu'il se déploie idéalement dans le cadre d'un colloque singulier favorisant une relation de confidence entre le soignant et le malade maintenu en chambre simple. Ainsi, c'est souvent pour des raisons organisationnelles que la personnalisation se déploie plus aisément dans les unités de soins palliatifs que dans les services 
de cancérologie, où elle ne peut être réalisée que de manière partielle.

\subsection{Accueillir}

L'accueil des patients dans les services observés tend à varier selon les modalités de leur entrée dans le service, selon qu'il existe, ou non, une relation suivie avec ces derniers et enfin, selon le type de service concerné. Lorsque des patients, méconnus du service, sont hospitalisés, les professionnels sont amenés à recueillir dans la précipitation les informations qui les concernent (parcours de vie et parcours de soin du patient, conscience qu'il a de sa situation, présence de proches disposés à participer aux soins, etc.). Les informations concernant l'histoire personnelle du malade, son rapport intimiste à la maladie ou à la douleur, la qualité de ses relations avec ses proches, tiennent une place particulièrement importante dans les unités de soins palliatifs ou pour les professionnels formés aux soins palliatifs. Ainsi, lors d'un transfert dans une USP, les malades peuvent être frappés, d'un lieu de soin à un autre, de constater autant de différences concernant les activités communicationnelles des professionnels.

Lorsque le patient est déjà connu des soignants du service auquel il accède, les modalités d'accueil se différencient puisque l'équipe dispose d'un certain nombre d'informations le concernant et qu'une relation de soin a déjà été mise en place avec lui. Les professionnels sont conduits à réamorcer la relation en discutant avec le malade des activités qu'il a effectuées à l'extérieur de l'établissement depuis son dernier séjour ou en lui demandant des nouvelles de ses proches.
Le retour des patients suivi antérieurement est généralement présenté comme une situation difficile, à la fois pour les malades et pour les professionnels (« lorsqu'ils reviennent, c'est que c'est mauvais signe », souligne une infirmière), nécessitant un réajustement du travail relationnel effectué jusqu'alors.

\subsection{Maintenir}

Lorsque le patient est exclusivement maintenu dans le service et que la durée de son séjour se prolonge ${ }^{14}$, des attentes vont se cristalliser sur le plan de ses conduites. Il est notamment invité par les professionnels $1^{\circ}$ ) à cultiver ses goûts et ses préférences dans les moments d'entre-deux (entre deux examens, traitements ou soins), $2^{\circ}$ ) à maintenir une ouverture à l'échange, $3^{\circ}$ ) à communiquer avec ses proches à propos de « choses importantes $», 4^{\circ}$ ) à régler avec l'aide des soignants les questions administratives et enfin $5^{\circ}$ ) à mettre en œuvre des « petits projets réalistes », notamment dans le cadre de sorties momentanées. Ce traitement social et moral de l'expérience du cancer, auquel les malades adhèrent peu ou prou $^{15}$, tend parfois à devenir une condition de leur maintien dans le service.

En outre, les malades maintenus en chambre double sont susceptibles, durant leur séjour, d'être transférés en chambre simple (altération corporelle, annonce d'une « mauvaise nouvelle» par le médecin, etc.) : un véritable « jeu de chaises musicales » peut se mettre en place dans le service. Dans les situations où l'état de santé du malade s'est rapidement dégradé (transformation brutale de l'apparence corporelle, troubles respiratoires, fortes douleurs, agitation, etc.), les proches sont 
prévenus par l'équipe avant le moment de la visite, que ce soit par téléphone ou bien à leur arrivée dans le service : il s'agit de désamorcer le choc émotionnel qui pourrait les affecter face au malade. Ce travail de préparation de l'entourage est parfois réalisé dans la précipitation, notamment dans les cas où de nouveaux symptômes apparaissent brutalement ${ }^{16}$.

\subsection{Transférer}

Les médecins et soignants s'efforcent au quotidien de trouver des solutions pour hospitaliser au sein d'un autre établissement les malades qu'ils ne peuvent accueillir ou garder dans le service. Les échanges téléphoniques auprès d'autres services conduisent les professionnels à user de tactiques pour obtenir l'approbation de leur interlocuteur (prendre sa « plus belle voix », comme le fait une infirmière, demander à s'entretenir avec un cadre ou un médecin que l'on connaît, mettre en avant des arguments, tel le jeune âge d'une patiente, pour convaincre de la nécessité d'une hospitalisation, etc.). Le travail relationnel à destination de malades inquiets, anxieux ou douloureux, maintenus à leur domicile, est également susceptible de se déployer à distance, par téléphone.

Le projet de transférer les patients à leur domicile exacerbe quant à lui les activités de surveillance des soignants vis-à-vis de l'entourage des malades, considéré en tant que « cheville ouvrière » du retour à domicile ${ }^{17}$. L'enjeu est ici de repérer si les proches sont disposés à assurer certains gestes de soins (aide pour la toilette et l'alimentation, massages, soutien affectif procuré au malade) et de surveiller la façon dont ils les réalisent. Parfois, il s'agit d' « éduquer » les proches pour réaliser des soins plus techniques (changement d'une canule, d'un pansement, gestion des pompes à morphine, surveillance des symptômes, etc.).

\section{La dynamique du travail relationnel au prisme des pressions organisationnelles : analyse croisée de deux études de cas}

La mise en perspective de deux études de cas permet de relever de façon plus précise les dynamiques du travail relationnel sous le jeu des contraintes organisationnelles. Ces études de cas se rapportent aux trajectoires de malades atteints d'un sarcome ayant été diagnostiqué à un stade avancé (présence de métastases). Il s'agit d'un homme et d'une femme présentant des situations sociales hétérogènes (milieu social, environnement familial et lieu d'habitation).

\section{1 $M^{m e}$ I., 44 ans, architecte, atteinte d'un sarcome avancé}

$M^{\text {me }}$ I. est âgée de 44 ans, elle est mariée, architecte, son époux est éditeur, leur fils est âgé de 12 ans, elle est atteinte d'un sarcome diagnostiqué il y a 18 mois, traité par radiothérapie. Elle a été hospitalisée en début de semaine en chambre double pour prise en charge de la douleur (J1). Après trois jours d'hospitalisation (J4), $\mathrm{M}^{\text {me }} \mathrm{I}$. ressent des fourmillements dans les jambes qui indiquent, d'après les médecins qui l'auscultent, une paralysie débutante du membre gauche. Ce symptôme précipite l'équipe dans un temps de l'urgence, tant sur le plan du travail médical que sur celui du travail relationnel. Le lendemain matin (J5), $\mathrm{M}^{\text {me }} \mathrm{I}$. reçoit des médecins du service l'annonce d'une paraplégie progressive 
et irrémédiable liée à l'évolution de son cancer. Elle fond en larmes, affirmant aux professionnels qu'elle ne souhaitait pas "mourir comme ça». En début d'après-midi, deux neurochirurgiens rendent visite à la patiente et, tout en l'informant de l'inefficacité de la radiothérapie, l'invitent à se prononcer sur son souhait de poursuivre les séances qui présentent toutefois des bénéfices en termes de contrôle de la douleur. La patiente, après avoir échangé avec son époux, décide de poursuivre le traitement.

L'équipe est alors confrontée à deux demandes de la part de $\mathrm{M}^{\mathrm{me}} \mathrm{I}$.. D'une part, celle-ci souhaite être transférée en chambre simple le plus rapidement possible. D'autre part, elle demande à retourner chez elle le week-end pour régler des questions administratives, ce qui suppose, au niveau du travail médical, une stabilisation de la douleur. Par ailleurs, elle réaffirme son souhait de regagner son domicile et de suivre les séances de radiothérapie en ambulatoire.

La première demande de la patiente (transfert en chambre simple) donne lieu à de nombreux échanges au sein de l'équipe. À ce moment-là, le service est « embolisé $»^{18}$ : aucune chambre simple n'est disponible et sept patients, dont certains sont maintenus à leur domicile, attendent depuis plusieurs jours d'être hospitalisés. Libérer une chambre ne peut être effectué qu'au détriment d'autres malades :

Infirmière (entrant dans la salle de transmission) : «Elle pleure $M^{\text {me }} I$., elle craque, elle veut une chambre seule».

Médecin : "Une chambre seule, c'est pas possible. À moins que $M^{\text {me }}$ K. et $M^{\text {me }} C$. soient dans la même chambre... mais ça me semble compliqué, $M^{m e} K$., elle est jeune (patiente âgée de 34 ans, atteinte d'un cancer avancé du col de l'utérus, en situation palliative). À vérifier si M. B. (patient agé de 32 ans, atteint d'une leucémie au stade terminal et pour lequel il existe un risque hémorragique) sort demain ... après, il faudrait pas qu'il saigne dans la nuit... » (Journal de terrain, réunion de transmission).

Deux conceptions antagonistes des soins sont alors débattues, la première consistant à considérer tous les patients, y compris ceux qui se trouvent en attente d'une hospitalisation, sur un même pied d'égalité (tous les patients sont malheureux, aucune demande ne devrait prendre le pas sur une autre), la seconde prenant appui sur la connaissance de la situation personnelle des malades et sur l'enjeu de maintenir une relation de confiance. Le psychologue du service, attentif aux échanges, souligne quant à lui les bénéfices qui pourraient être obtenus sur le plan du « travail de deuil » de la patiente, dans le cas où la réponse de l'équipe serait différée : « (...) il vaudrait mieux attendre demain. Si on répond de suite, elle aura peut-être les mêmes attentes par rapport aux traitements. Il faut laisser s'exprimer sa tristesse ».

Après avoir été transférée à son domicile durant le week-end, $\mathrm{M}^{\mathrm{me}} \mathrm{I}$. est de retour dans le service (J8). Les soignants constatent avec surprise que la patiente et son époux sont calmes et souriants. Ce constat est également relevé par les professionnels de l'HAD qui ont rencontré le couple dans le service le mardi (J9), ceci en vue de préparer une hospitalisation à domicile pour le mercredi (J10).

Médecin HAD : «C'est ahurissant comme son 
mari contrôle tout... je sais pas comment on va faire... ».

Médecin du service : «Elle s'est dégradée très rapidement la semaine dernière... le jeudi son mari a dit qu'elle demanderait l'euthanasie. Elle a été dans la colère pendant 48 heures et depuis le début de la semaine elle est souriante, calme, le mari aussi. (...) Il y a une acceptation rapide et surprenante d'elle et de son époux, alors qu'il y a une évolution brutale de la maladie ». (Journal de terrain, réunion de coordination en soins palliatifs).

\subsection{S., 50 ans, chauffeur-routier, sarcome avancé}

M. S., âgé de 50 ans, est chauffeur-routier, son épouse est femme au foyer, ils ont trois enfants. Son cancer a été diagnostiqué quatre mois auparavant, il a été hospitalisé au service des urgences suite à la demande de sa fille pour de fortes douleurs à l'épaule, puis transféré dans un établissement de traitement des cancers. Dès le diagnostic, l'épouse et les enfants de M. S. insiste auprès de lui pour qu'il accepte une opération chirurgicale. Les médecins relèvent que le patient est en dépression majeure, ce dernier exprimant à plusieurs reprises le souhait de mettre fin à ses jours. Quatre mois après le diagnostic, M. S. est transféré un samedi depuis un autre service de l'établissement pour altération de l'état général et douleurs (J1). Il est étiqueté « soins palliatifs » par l'équipe dès le lundi suivant (J3). Le lendemain (J4), les médecins discutent de la possibilité de le transférer dans un service de suite et de réadaptation, option qui sera rapidement abandonnée. L'épouse du patient est considérée par les soignants comme étant « très demandeuse » d'informations médicales, voire « envahissante». Des problèmes sont également identifiés par les soignants sur le plan de la gestion de la douleur, le patient présentant des difficultés à l'auto-évaluer.

Le lendemain, le projet d'une hospitalisation à domicile est envisagé par les médecins (J5). Au bout de 10 jours, à ce projet s'ajoute l'alternative d'un transfert vers une USP située proche du domicile du patient. À J13, l'équipe coordinatrice du service HAD rapporte au médecin du service que : «M. S. est pas du tout prêt pour l'HAD. Ils sont tous trop désespérés, il peut même pas y réfléchir. Il dit qu'il est bien chez vous. Il touche $d u$ bois pour pas sortir " (Médecin HAD). Le médecin du service précise alors qu'il existe un problème avec le médecin référent du patient «qui ne dit pas trop les choses » quant à l'évolution de la maladie, ce qui contribue à ses yeux à bloquer la possibilité d'un transfert vers un autre lieu de soin.

Les semaines passent, plusieurs protocoles de traitement de la douleur se succèdent, une radiothérapie à visée antalgique est programmée. L'équipe s'interroge sur les conduites du patient hospitalisé, considérées comme étant « étranges » (infirmières) ou " inadaptées 》 (algologue, psychologue). Les proches sont suspectés de dissimuler au patient le pronostic. Cependant, aux yeux des professionnels, M. S. « se sent bien » dans le service, il le dit. Il commence également à « poser des questions » aux soignants et à s'interroger sur son devenir. Le travail relationnel de l'équipe s'oriente autour de l'enjeu d'amener progressivement M. S. à accéder à une conscience ouverte du pronostic, ce qui nécessite de le maintenir dans le service 
quelques jours supplémentaires et de négocier son transfert pas à pas.

Des contingences organisationnelles vont finalement accélérer le transfert de M. S. en USP, qui sera effectué deux jours plus tard, suite à l'appel d'un établissement annonçant qu'une chambre venait de se libérer.

Médecin 1 : «Ce matin, je lui ai dit qu’il partait dans une USP».

Interne : «Oui, il a dit à une infirmière : "Si je pars dans une USP, c'est que je suis fichu et qu'ils veulent plus me garder »».

Médecin 1 : «En même temps, c'est bien de lui avoir dit... depuis qu'il est là, il a pas avancé, il avance pas ».

Médecin 2: «Oui, c'est bien, ça le fait avancer... ». (Journal de terrain, couloir du service).

\subsection{Des capacités de négociation hétérogènes face aux professionnels}

Ces deux études de cas présentent un certain nombre de points de clivages. En premier lieu, elles se différencient sur le plan du contrôle exercé par les malades sur la trajectoire du cancer (contrôle des informations médicales, engagement dans les décisions concernant les traitements et les soins, leur durée, les lieux de prise en charge). Ensuite, elles se distinguent sur le plan de la relation aux professionnels : si les soignants s'efforcent de faire en sorte que $\mathrm{M}^{\mathrm{me}} \mathrm{I}$. " lâche prise » vis-à-vis des soins proposés et évacue des émotions «négatives », dans le cas de M. S., tout l'enjeu réside dans celui de responsabiliser le patient afin qu'il soit plus impliqué dans les décisions. Le positionnement de l'entourage des malades est également contrasté. Dans le premier cas, la patiente et son époux s'accordent pour faire front à l'autorité des professionnels et s'efforcent de préserver leur marge de manœuvre dans la négociation des soins. Dans le second, on observe des conduites divergentes entre M. S. et ses proches (patient faiblement engagé dans la quête des informations médicales, contrairement à son épouse). Cette situation a pour effet de réduire les capacités de négociation de M. S. face aux professionnels. Sur le plan du travail relationnel des soignants, alors que diverses pressions morales se déploient (qualification d'une « crise suicidaire » dans les deux cas, « violence » de l'annonce du pronostic ou de l' « abandon » du malade, etc.), c'est le poids des contraintes organisationnelles (embolie du service) qui va finalement faire flancher la balance vers un travail de responsabilisation des patients, nécessaire à leur transfert vers un autre lieu de soin.

\section{Conclusion}

Le retour du « sujet de soin » dans la médecine et, plus largement, dans la vie ordinaire, a donné lieu à de nombreux travaux en sciences sociales et en sciences de la santé ${ }^{19}$. Cette contribution se démarque de certains d'entre eux dans le sens où il ne s'agit pas tant de discuter des conceptions philosophiques ou juridiques du « sujet de soin » que de l'appréhender en tant que norme sociale et morale orientant le travail relationnel des soignants. L'observation de ce travail indique clairement qu'il s'agit d'une norme flexible, variant au fil des trajectoires spatio-temporelles du cancer, des interactions soignant-soigné, de la confiance accordée aux proches du malade et enfin, comme il a été indiqué, variant sous le jeu 
de contingences organisationnelles au sein des services hospitaliers. Dans le service observé, il apparaît en effet que la responsabilisation des malades tend à s'accroître dans les situations de fortes pressions organisationnelles (nécessité de libérer des chambres, lourdeur des soins techniques, etc.) et au détriment du modèle de la personnalisation qui est pourtant celui valorisé par l'équipe ${ }^{20}$. Or, lorsque le travail relationnel de personnalisation est abandonné, l'épuisement de ce modèle est susceptible d'être reporté sur les conduites du malade ou sur celles de ses proches (insuffisance du «travail sur soi », « déni » de l'entourage). C'est sans doute à cet endroit que la responsabilisation prend tout son sens, puisqu'elle conduit les professionnels à désigner la responsabilité des malades et de leur entourage dans les enkystements du travail relationnel.

Il apparaît toutefois que dans certaines situations, les contraintes organisationnelles permettent au malade et à ses proches de disposer d'une marge de manœuvre dans le contrôle qu'ils effectuent sur la trajectoire de maladie. Ainsi, dans le cas de $\mathrm{M}^{\mathrm{me}} \mathrm{I}$, une alternance entre maintien à domicile et retour dans le service, souhaitée par la patiente et par son époux, favorise la mobilisation de ressources et la mise en œuvre de tactiques qui lui permettent de négocier avec les professionnels et de défendre, voire parfois d'imposer son point de vue (en particulier à son domicile). Cet engagement dans les décisions et cette puissance d'action de la patiente sont d'autant plus prégnants qu'ils sont encouragés et relayés par son conjoint. Dans une tout autre situation, où un moindre engagement du patient dans les décisions et les soins et où des discordances avec ses proches ont pu être observées (Mr S.), le transfert vers un autre établissement est associé à une perte de contrôle sur la trajectoire de maladie.

Aussi, ces observations conduisent à quitter momentanément la scène de l' " hôpital sous pression $\gg{ }^{21}$ pour investir les autres lieux de soins dans lesquels les malades atteints de cancer avancé sont susceptibles de circuler de gré ou de force. Ce faisant, il s'agit de saisir les trajectoires circulatoires dessinées par les malades à la recherche du « meilleur soin possible », celles qu'ils négocient auprès de leur médecin traitant, auprès des spécialistes ou en mobilisant leur propre réseau de relations, celles enfin qu'ils subissent dans les aléas des pressions organisationnelles affectant les services hospitaliers. Les trajectoires de cancer avancé tendent alors à prendre une forme itinérante pour laquelle des inégalités sociales dans l'accès aux soins et des différenciations sociales dans les usages des lieux de soins se retrouvent de manière exacerbée. 
$\mathrm{N} \cdot \mathrm{O} \cdot \mathrm{T} \cdot \mathrm{E} \cdot \mathrm{S}$

1. Notamment le Plan cancer 2009-2013.

2. Ces conflits latents se rapportent principalement au rapport du patient à l'information médicale, à son suivi des traitements et des soins, à la gestion de la douleur en tant que souffrance physique et psychique et enfin à la conscience du malade quant au pronostic.

3. Voir H. Marche, « Du curatif au palliatif dans un service de cancérologie : la gestion sociale des incertitudes » in S. Pennec (dir.), Des vivants et des morts. Des constructions sociales de la « bonne mort », Brest, ARS-CRBC, UBO, 2004, pp. 143-152.

4. La gestion de cette incertitude implique l'appréhension des différentes phases de la maladie (rémission, récidive, évolution, etc.) et la mise en place du traitement. Voir M. Ménoret, Les temps du cancer, Paris, CNRS éditions, 1999. Voir également I. Baszanger, « Entre traitement de la dernière chance et palliatif pur : les frontières invisibles des innovations thérapeutiques », Sciences Sociales et Santé, vol.18, n², pp. 67-94, juin 2000.

5. Pour les phases très avancées de cancer, ces questionnements se centrent principalement sur la question du lieu du mourir (Baszanger, 2000, op. cit.).

6. Des observations ont également été menées au sein d'une clinique (unité de soins palliatifs) et auprès d'équipes d'hospitalisation à domicile. L'enquête a été réalisée au cours d'une recherche post-doctorale CNRS (2010-2013) dans le cadre d'un programme financé par l'Institut National du Cancer (convention 2010-040) et la Fondation de France (Engt 2009 005989).

7. Dans ce service, les malades étiquetés « soins palliatifs » sont ceux pour lesquels il n'existe plus de traitements spécifiques et dont l'espérance de vie est estimée inférieure à trois mois. L'étiquetage de ces patients donne souvent lieu à des désaccords au sein de l'équipe ou entre médecins du service et médecins référents.

8. Une soixantaine d'entretiens a été réalisée.

9. Tout en empruntant une perspective en terme de « trajectoire » (voir A. Strauss, « Maladie et trajectoires » in La trame de la négociation - Sociologie qualitative et interactionniste, Paris, L'Harmattan, 1992 [1985], pp. 143-175 ; Ménoret, 1999, op.cit.), l'analyse des données a conduit à examiner la façon dont les professionnels qualifient les émotions des malades, ceci afin de mieux comprendre le travail relationnel qui en découle.

10. N. Elias, La société des individus, Paris, Fayart, 1991.

11. P. Pinell, Naissance d'un fléau, histoire de la lutte contre le cancer en France, 1890-1940, Paris, A.M. Métailié, 1992 ; H. Marche, « Le contrôle ordinaire et institutionnel de l'expérience du cancer : les limites $\mathrm{du}$ « patient-sentinelle » » in F. Douguet, F. LeborgneUguen, S. Pennec (dir.), Soins négociés. Ordre-s et désordre-s des négociations, Rennes, PUR, 2013 [à paraître].

12. Sans entrer ici dans le détail, il importe de préciser que le travail relationnel correspond à un éventail de postures professionnelles, celui d'un médecin étant plus étendu que celui d'un agent des services hospitaliers.

13. Voir ma thèse : H. Marche, Le travail relationnel au cour de l'expérience du cancer. Mises en forme ordinaires et institutionnelles de cette épreuve, thèse de doctorat de sociologie sous la direction de Marcel Drulhe, Université Toulouse 2, juillet 2009.

14. Paradoxalement, les cas de longues hospitalisations remettent en question les postures adoptées par les professionnels, qui peuvent être conduits à « dépersonnaliser » la relation de soin (tourner lors des soins, éviter d'aborder avec le patient des thématiques relatives à sa vie privée ou de partager avec lui des aspects de la vie non-professionnelle) afin que l'accompagnement 
du malade ne devienne chronophage ou épuisant.

15. Ces attentes professionnelles n'excluent pas des « résistances » de certains malades à y répondre, ou encore des capacités à négocier auprès des soignants un déplacement du travail relationnel.

16. Les décès par hémorragie interne, non prévus par l'équipe, sont des situations pour lesquelles les soignants n'ont pas eu le temps d'effectuer le travail de préparation des proches (travail sur la conscience du pronostic, procédures administratives, accompagnement émotionnel en vue de favoriser un « travail de deuil », etc.).

17. Pour les professionnels, une des premières conditions du transfert à domicile est celle de l'engagement des proches dans les soins et leur disponibilité auprès $\mathrm{du}$ malade (ces attentes se reportent principalement sur les membres du groupe familial et en particulier sur les femmes).

18. Ce terme d'embolie, issu du langage médical, est mobilisé par les médecins et soignants pour signifier un « blocage » de nombreux malades au sein du service et une impossibilité d'accueillir de nouveaux patients. Un médecin travaillant dans une unité de soins palliatifs a également mobilisé l'expression «le patient thrombose un plumard » pour signifier qu'un malade « bloque » un lit dans le service.

19. Sans lister de manière exhaustive, voir les travaux issus des théories du care (P. Molinier, S. Laugier, P. Paperman, Qu'est-ce que le care? Souci des autres, sensibilité, responsabilité, Paris, Payot et Rivages, 2009), les recherches autour de l'importance de la subjectivité du patient dans les soins (C.-O. Doron, C. Lefève, A.-C. Masquelet, «Introduction » in Soin et subjectivité, Paris, PUF, coll. « Science, Histoire et Société », 2011, pp. 3-11), celles issus de la sociologie de l'éthique biomédicale, etc.

20. Et par l' « esprit » des soins palliatifs en général. Voir H. Marche, 2009, op. cit.
21. N. Belorgey, L'hôpital sous pression. Enquête sur le nouveau management public, Paris, La Découverte, 2010. 


\title{
$R \cdot E ́ \cdot S \cdot U \cdot M \cdot E ́$
}

Ce texte vise à éclairer les variations du travail relationnel des professionnels auprès des malades atteints de cancer avancé, dans un contexte de réduction des séjours hospitaliers et de rationalisation des soins. En prenant appui sur une enquête ethnographique dans des services de cancérologie et de soins palliatifs, l'article indique en quoi la norme du malade en tant que « sujet » mobilisée par les soignants tend à varier selon les contextes de soin. La mise en perspective de deux études de cas permet de rendre compte du poids des pressions organisationnelles sur le travail relationnel, induisant un déplacement de la " personnalisation » des soins vers une « responsabilisation» des malades et de leur entourage.

\begin{abstract}
The aim of this paper is to clarify the changes in relationships work between the professionals and the patients with advanced cancer, in a context of reduced hospital stays and rationalization of care. Drawing on an ethnographic study in cancer services and palliative care, the article shows how the patient's norm as a « subject » and « person » mobilized by caregivers tends to vary according to the context of care. The comparison of two case studies helps to account for the weight of organizational pressures on the relational work, inducing a shift in the "personalization » of care towards a « responsabilization» for patients and their lay caregivers.
\end{abstract}

